\title{
REVIEW OF CATHETER VENOGRAPHY PROTOCOLS IN THE EVALUATION OF CHRONIC CEREBROSPINAL VENOUS INSUFFICIENCY
}

\author{
Hector Ferral \\ Department of Radiology, NorthShore University HealthSystem, Evanston, USA
}

\begin{abstract}
Catheter venography has been considered to be the "gold standard" in the evaluation of venous pathology. Chronic cerebrospinal venous insufficiency (CCSVI) has recently been described as a condition characterised by the presence of multiple stenotic lesions of the main pathways of extracranial venous drainage, especially the internal jugular veins and the azygos vein. The primary screening diagnostic method to identify the venous lesions has been Doppler sonography. Once the abnormalities are identified by the non-invasive method, patients undergo catheter venography to confirm the presence of venous lesions with the aim of performing treatment, which has mainly been balloon angioplasty. Since the inception of CCSVI, several venographic protocols to evaluate the internal jugular and azygos veins have been described, but none of them have been scientifically validated. The purpose of this review is to analyse the most recent catheter venography protocols reported for the diagnosis and management of CCSVI. The materials and methods sections of the most recently published series describing catheter venography for the evaluation and management for CCSVI were reviewed. The technical details, including venous access selection site, catheter selection, ideal catheter position, contrast injection techniques, and interpretation of venographic findings, were reviewed. Comments on each of the technical factors are presented. Final comments with proposed techniques are presented.
\end{abstract}

Key words: chronic cerebrospinal venous insufficiency, catheter venography, internal jugular veins.

\author{
REVIEW PAPER
}

Phlebological Review 2014; 22, 1: 11-17

DOI: 10.5114/pr.2014.46048

Submitted: 4.06 .2014

Accepted: 7.09 .2014

\section{ADDRESS FOR CORRESPONDENCE:}

Dr. Hector Ferral, Department of Radiology, NorthShore University HealthSystem,

2650 Ridge Avenue, 60201 Evanston, USA, e-mail: hectorferral@gmail.com

\section{INTRODUCTION}

Catheter venography has been considered to be the "gold standard" in the evaluation of venous pathology [1-4]. Venography provides valuable information regarding stenotic areas, flow patterns, reflux, and the presence of collaterals [2]. Chronic cerebrospinal venous insufficiency (CCSVI) is a venous vascular condition recently described by Zamboni et al. [5]. These investigators described CCSVI as a syndrome characterised by the presence of multiple stenotic lesions of the main pathways of extracranial venous drainage, especially the internal jugular veins and the azygos vein [5]. According to these authors, the vascular occlusive lesions characteristic of CCSVI are truncular malformations that can be clearly demonstrated by selective catheter venography [5]. Since the inception of CCSVI [5], several venographic protocols to evaluate the jugular and azygos veins have been described in recent publications $[1,4$, 6-12]; however, none of these have been validated, and to the present time there is no venographic protocol that has been accepted as a standard in the evaluation of this condition $[1,12]$.

Interestingly, the anatomy, haemodynamics, and flow physiology of the neck and central veins are poorly under- stood and thus, the interpretation of catheter venography may have limitations $[1,10,13]$; this may be part of the reason why diagnostic catheter venography protocols for CCSVI have been so variable. The purpose of this article is to review in detail the venographic protocols described for the evaluation of CCSVI in recently published series and to propose a reasonable venous evaluation protocol that may be used as a "standard" technique for future reference and publications.

\section{CATHETER INSERTION SITE SELECTION}

Zamboni et al. described the selective catheter venographic technique for the evaluation and management of patients thought to have CCSVI [5]. These authors elected to use the left common femoral vein for access to perform selective venography of the lumbar, left renal, azygos, and internal jugular veins. Intervention was only performed in the jugular and azygos veins [5]. Traboulsee et al. and Veroux et al. exclusively used the right femoral vein $[4,12]$. In other series, the access site was not specified $[7-9,14]$. The advantage of using the left femoral vein is the ability to evaluate the left iliac vein and the ascending lumbar veins; however, since these veins are not treated, some operators prefer the right femoral vein becuase 
it facilitates access to the internal jugular veins and azygos veins, which are the treatment target veins [1].

Comment: From a practical standpoint the use of the right common femoral vein probably makes more sense since intervention is not performed in either the left iliac or lumbar veins.

\section{CATHETER SELECTION}

Most authors use an end-hole catheter to perform the jugular and azygos venography $[4,5,7-9,12,14]$. When using an end-hole catheter, it is important to rotate the tip so it does not engage a collateral branch, giving a false impression of excessive collateral flow after injections $[1,4,14]$. Ferral et al. performed the diagnostic venography with a straight catheter with side holes, avoiding the potential problem of directly catheterising a collateral vein [6]. The disadvantage of this technique is that it requires catheter exchanges every time a diagnostic venogram is performed [6].

Comment: An excellent alternative would be the use of a custom-made angled catheter with 2-3 side holes.

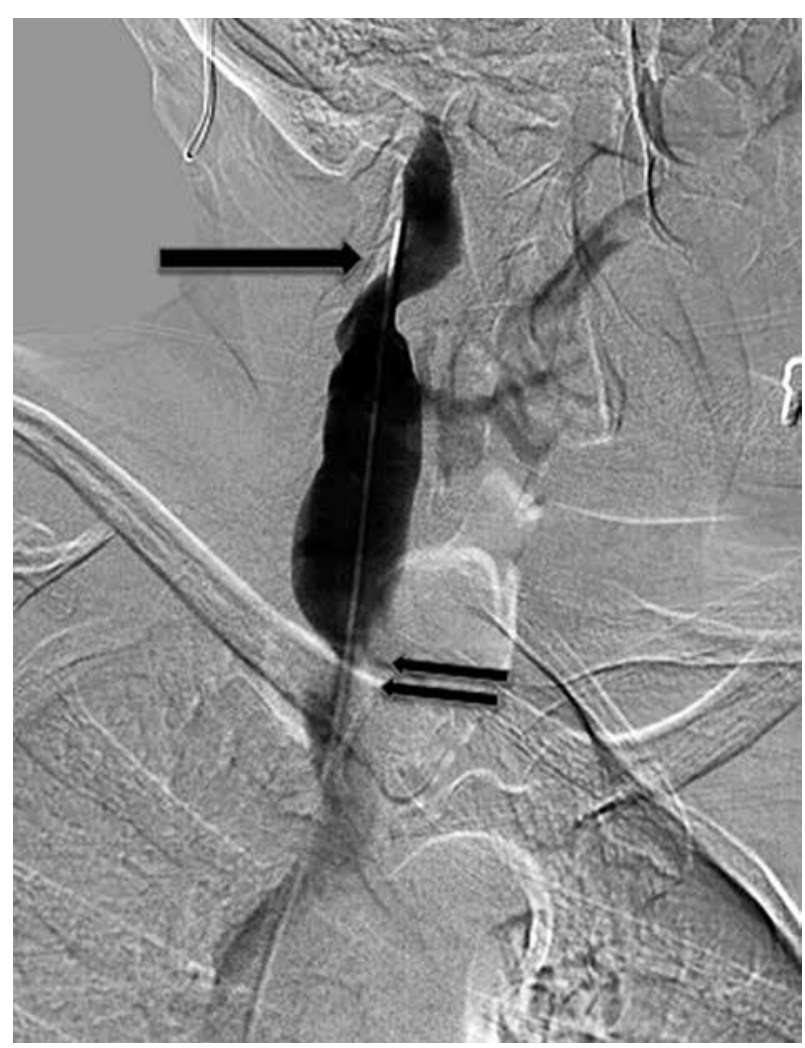

Fig. 1. Diagnostic internal jugular venogram

The figure shows an image selected from a digital subtraction venogram of the right internal jugular vein. The injection was performed using a low-pressure power injector. The total injected volume was $15 \mathrm{ml}$ at a rate of $5 \mathrm{ml} / \mathrm{s}$. Notice the tip of the multisidehole catheter, placed at the skull base (large arrow). The patient's head is slightly turned to the left side and the image intensifier has a 25 right anterior oblique projection. An internal jugular venous valve malformation is clearly demonstrated (double arrow). There is minimal opacification of collaterals.

\section{CATHETER POSITION}

The ideal position of the angiographic catheter within the jugular and azygos veins to obtain an ideal venogram is still a matter of debate. Most authors placed the venographic catheter at the skull base to perform the diagnostic jugular venograms $[4,6-8,12]$ (Fig. 1). Ludyga et al. placed the angiographic catheter in three locations within the jugular veins to perform the diagnostic venograms: at the foramen jugulare, at the level of the junction with the facial vein, and slightly cranial from the valve in the junction with the brachiocephalic vein [14]. Other authors did not specify catheter position within the jugular veins $[5,9]$.

Regarding the evaluation of the azygos vein, Zamboni et al. placed the angiographic catheter deep in the azygos vein, close to the confluence with the hemiazygos vein [5]. Most authors place the azygos vein catheter in this same position $[1,6,8]$.

Comment: Probably the best place for a diagnostic catheter for jugular vein evaluation is the skull base as this position is easily obtained and reproducible. Regarding the azygos vein, there is little debate on the catheter position deep in the azygos vein, close to the confluence with the hemiazygos vein.

\section{CONTRAST INJECTION}

Contrast can be injected as a full-strength solution or a 50\%-diluted solution with normal saline. Regarding the injection technique, it can be performed either by manual or power injection [1]. These technical factors of the diagnostic venographic technique are important and should be documented in any manuscript on the topic.

Authors who advocate injection of full strength contrast argue that full strength allows better visualisation of collateral pathways in patients with venous obstructions [1]. When contrast media is diluted for digital subtraction imaging, it is usually diluted to $50 \%$ contrast, $50 \%$ normal saline. Dilution of contrast allows the operator to identify subtle findings that would otherwise be lost within the dark density of full-strength contrast and still allows visualisation of reflux and collateral pathways [6] (Figs. 2 and 3). Using diluted contrast medium also offers the added advantage of reduced contrast load per procedure. Based on the information reported in recent publications, authors who advocate venography as the ideal single imaging method to evaluate CCSVI support the use of diluted contrast $[1,14]$. The importance of multimodal imaging to evaluate the jugular and azygos veins has been emphasised in recent reports [11]. The ideal endovascular modality to complement venography is intravascular ultrasound (IVUS) $[6,15]$. The use of IVUS allows visualisation of subtle endoluminal lesions $[6,7,15]$ and would avoid the need for detailed, dilute contrast venography; however, IVUS is not always available in every angio- 

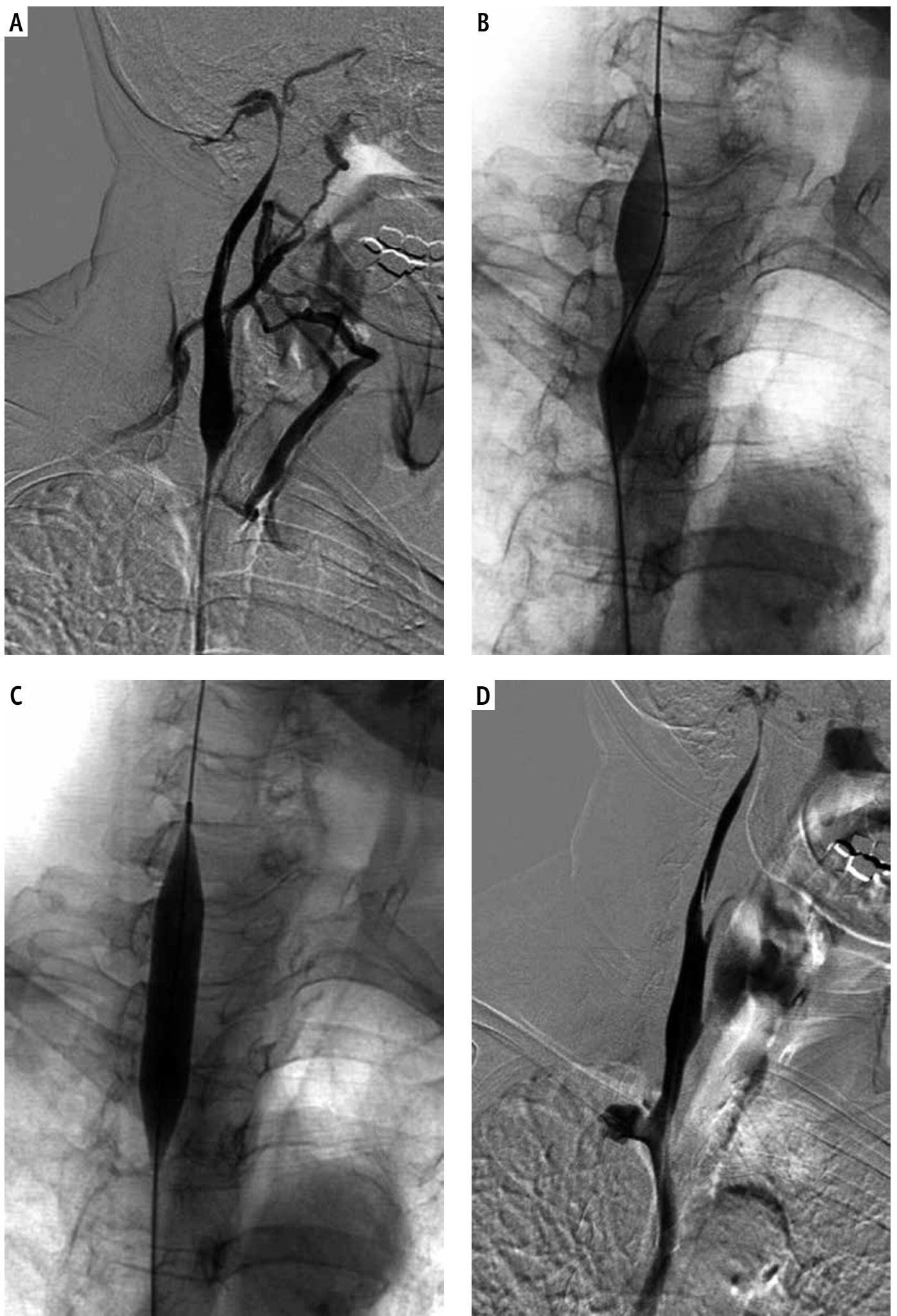

Fig. 2. Internal jugular venogram with a power injector

A) Selective right internal jugular venogram. A straight multisidehole catheter was placed at the skull base. Low-pressure power injection of $15 \mathrm{ml}$ of $50 \%$ diluted contrast at $5 \mathrm{ml} / \mathrm{s}$ show a venous valve malformation of the right internal jugular vein and opacification of collaterals.

B) Spot film obtained during balloon angioplasty shows the waist at the site of venous valve malformation.

C) Spot film obtained during balloon angioplasty shows complete effacement of the waist previously seen at the site of venous valve malformation.

D) Selective right internal jugular venogram after angioplasty, using the same injection parameters now shows a patent right internal jugular vein with no opacification of collaterals. Power injection in this case does not result in "false" opacification of collaterals. 

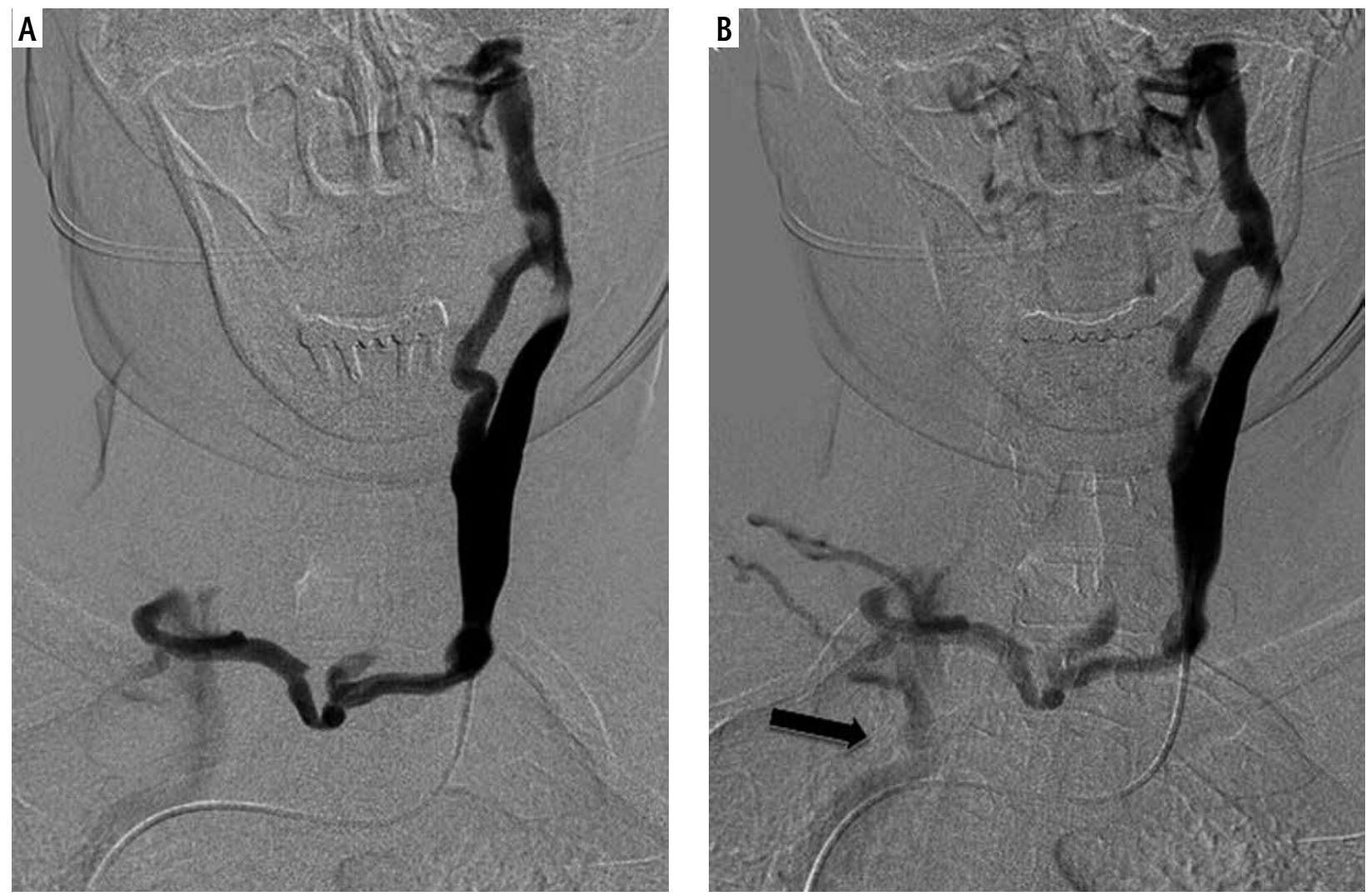

Fig. 3. Left internal jugular venogram: delayed emptying time

Figures A and B were obtained with the patient's head in neutral position. The straight multisidehole catheter was placed at the skull base. The contrast was injected using low-pressure power injection of $15 \mathrm{ml}$ of $50 \%$ diluted contrast at a rate of $5 \mathrm{ml} / \mathrm{s}$.

Figure $\mathbf{A}$ shows a severe stenosis of the left internal jugular vein secondary to a venous valve malformation. There is opacification of large collaterals contrast flowing to the contralateral jugular vein via the thyroidal plexus.

Figure $\mathbf{B}$ is a later frame obtained $4 \mathrm{~s}$ later and shows faint opacification of the contralateral jugular vein and right innominate vein (arrow). Notice that the most central segment of the left internal jugular vein is still not opacified. The use of diluted contrast still demonstrates the opacification of collaterals.

graphic suite and furthermore, not all operators are comfortable with the interpretation of IVUS images.

Regarding to the use of manual injections versus power injections, some authors advocate the use of manual injections to avoid excessive pressure during injections, thus avoiding the unnecessary or "false" opacification of collaterals $[8,9,14]$. Other authors advocate the use of a power injector, with specific volumes of contrast and flow rates. The total volume injected per site is usually in the range of 8-15 $\mathrm{ml}$ at flow rates ranging between 2 and $5 \mathrm{ml} / \mathrm{s}[1,4-6,12]$ (Fig. 2).

Comment: Dilute contrast venography probably offers more advantages than venography using full strength contrast. The use of power injection has the advantage of making venous evaluation protocols reproducible and allows for objective evaluation of contrast emptying times, as will be discussed later $[1,4,7,12]$.

\section{INTERPRETATION OF VENOGRAPHIC FINDINGS}

Zamboni's original publication called for evaluation of the lumbar, left renal, azygos, and internal jugular veins [5]. Most authors have focused on the evaluation of the internal jugular and azygos veins since intervention is almost exclusively performed in these veins [4]. The primary objective of the venographic evaluation in these patients is to detect lesions that need to be treated, i.e. lesions that are potentially haemodynamically significant. Parameters that have been evaluated during the venographic examination of the jugular and azygos veins include: percentage of stenosis, lesion morphology, presence of reflux, opacification of collaterals, pressure gradients, and delayed emptying of the injected contrast.

Most authors agree that a significant stenosis is present when there is a venous luminal reduction greater than $50 \%[1,4-6,8,9,12]$; however, as mentioned by Simka, this is an extrapolation from arterial work and more research may be necessary to validate this concept before it can be applied to the evaluation of venous pathology [1].

Regarding the morphology of the lesions, only Zamboni et al. explore these in detail and describe six main malformations [5]: 
- annulus: significant circumferential stenosis of the whole venous wall,

- septum/valve malformation: anomalous valve apparatus causing significant flow obstacles at the level of the internal jugular vein junction with the brachiocephalic trunk (Figs. 1-3),

- hypoplasia: underdeveloped long venous segments,

- twisting: stenosis secondary to a twisted venous segment (Fig. 4),

- membranous obstruction: membrane almost occluding a vein,

- agenesis: complete anatomic absence of a venous segment.

The presence of reflux has been addressed by several authors $[6,9,14]$; Ludyga et al. proposed a classification of venographic findings based on reflux patters [14]. These authors proposed four grades as follows:

- grade 1: venous outflow slowed down, no reflux detected,

- grade 2: venous outflow slowed down, mild reflux and / or pre-stenotic dilatation of the vein,

- grade 3: venous outflow slowed down with reflux and outflow through collaterals,

- grade 4: no outflow through the vein, huge outflow through collaterals [14].

Karmon et al. classified the presence of collaterals as either epidural plexus or other collaterals, not along the spinal cord, and paravertebral as well as deep cervical veins [7]. The degree of opacification was classified as scores of 1-3, with 1 being early filling of spinal epidural collaterals and 3 suggesting marked opacification of the spinal epidural collaterals in both early and late phases and extending throughout the cervicothoracic spinal canal. A score of 2 is an intermediate stage between the two previously mentioned [7]. Azygos vein collaterals were classified in a similar fashion [7].

Traboulsee et al. classified collaterals as abnormal if there were one or more vessels greater than $50 \%$ the size of the adjacent primary vessels or two or more collaterals vessels present at less than $50 \%$ of the size of the adjacent primary vessel [4].

The measurement of pressure gradients across the stenotic areas in the jugular and azygos veins is still a matter of debate. Zamboni et al. performed contrast venography in 65 patients with clinically defined multiple sclerosis who met non-invasive criteria for CCSVI and 48 control patients who had no neurological disorder but required venography for other reasons [16]. Zamboni reported significant pressure differences across stenotic segments in the azygos and jugular veins; comparing pressures in these veins with inferior vena caval pressures [16]. On the other hand, other operators who have performed
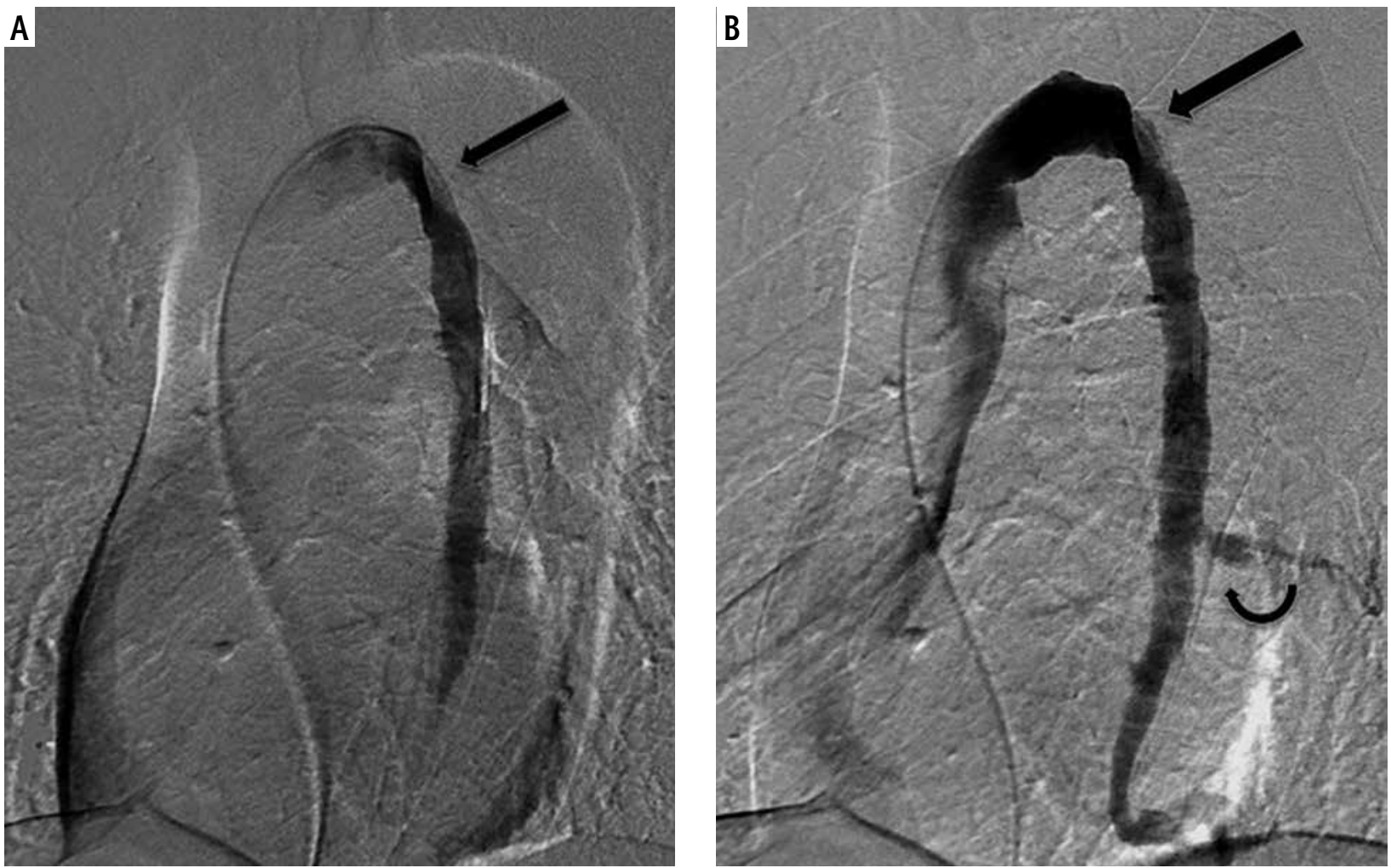

Fig. 4. Azygos venogram: twisting lesion and delayed contrast emptying

Figures $\mathbf{A}$ and $\mathbf{B}$ were obtained during a selective digital subtraction azygos venogram. The image intensifier was angled at 55 left anterior oblique projection. The straight multisidehole catheter is placed deep into the azygos vein. A low-pressure power injection of $15 \mathrm{ml}$ of $50 \%$ diluted contrast at $5 \mathrm{ml} / \mathrm{s}$ was performed. Figure $\mathbf{A}$ shows a twisting lesion of the azygos vein just caudal $t$ the arch (arrow).

Figure $\mathbf{B}$ was obtained 5 seconds later and shows persistence of the twisting lesion (straight arrow), delayed contrast emptying and opacification of epidural collaterals (curved arrow). 
Table 1. Venographic technique: comparative table

\begin{tabular}{llllllc}
\hline Author & Year & Access & Catheter & Position & Injection & Contrast \\
\hline Zamboni & 2009 & LFV & end hole & NS & power & full \\
\hline Ludyga & 2010 & NS & end hole & multiple & hand & diluted \\
\hline Mandato & 2012 & NS & end hole & NS & hand & diluted \\
\hline Hubbard & 2012 & NS & end hole & SB & hand & NS \\
\hline Ferral & 2013 & LFV & side hole & SB & power & diluted \\
\hline Karmon & 2013 & NS & end hole & SB & power & NS \\
\hline Traboulsee & 2013 & RFV & end hole & SB & power & NS \\
\hline Veroux & 2013 & RFV & end hole & SB & power & full \\
\hline Simka/ISNVD & 2013 & RFV & NS & NS & power & either \\
\hline
\end{tabular}

LFV - left femoral vein; RFV - right femoral vein; NS - not specified; SB - skull base

pressure gradient measurements have failed to document significant differences in pressures across these stenotic lesions [6] and for this reason, venous pressures have not been routinely measured in these procedures $[4,6,9,12$, 14]. A possible explanation for this phenomenon is that the pressure gradient is split within the large collateral network seen in these patients.

In a later report, Zamboni et al. measured venous pressures before and after treatment within the jugular and azygos veins [5]. These authors did not find significant pressure differences between jugular veins considered to be normal and jugular veins found to be stenotic [5]; however, they did find a significant reduction in pressures (measured in $\mathrm{cm} \mathrm{H}_{2} \mathrm{O}$ ) within the treated veins after successful treatment [5]. The issue of pressure measurements has not been solved, but it would probably make sense to add pressure measurements to all future reports.

Delayed emptying of the injected contrast has been described in more detail in recent reports $[4,7,12]$. Traboulsee described stasis as the presence of contrast within the injected vein for more than $4 \mathrm{~s}$ after injection [4]. Karmon described the contrast emptying time (ET) as the duration of clearance of all contrast material from the vein being studied after completion of contrast injection. These authors used manual injections in the first part of their study (PREMISE) and power injections in the second part, which included 20 patients [7]. These authors performed four second injections in the jugular veins (12 $\mathrm{ml}$ total volume at a rate of $3 \mathrm{ml} / \mathrm{s}$ ) and three second injections in the azygos veins $(9 \mathrm{ml}$ total volume at $3 \mathrm{ml} / \mathrm{s}$ ) and diagnosed the ET as prolonged if contrast remained within the injected vein for longer than $6 \mathrm{~s}$ [7]. These authors found correlation between the emptying time and stenotic segments. Stenotic veins showed ET greater than $6 \mathrm{~s}$, and normal veins had ET of less than 6 s [7].

Veroux described a detailed venographic protocol that included low-pressure contrast administration using a power injector. The total volume used by these authors was $8 \mathrm{ml}$ injected at a rate of $4 \mathrm{ml} / \mathrm{s}$. Image acquisition was at a rate of three frames per second for the first $4 \mathrm{~s}$ and then two frames per second for $8 \mathrm{~s}$. The patients were asked to breathe normally during image acquisition and maintain the head in a straight position. The clearance time of contrast after injection was calculated using receiver operating characteristic analysis, and the authors found that $4 \mathrm{~s}$ was the maximal cutoff that gave the highest sensitivity and specificity for normal clearance. Thus, these authors concluded that in the circumstances described, contrast emptying or clearance of less than $4 \mathrm{~s}$ was considered to be normal [12], patients with a clearance time between 4 and $6 \mathrm{~s}$ were considered to have moderate delayed flow, and patients with clearance greater than $6 \mathrm{~s}$ were considered to have severe delayed flow [12]. These authors only treated veins that showed either moderate delayed flow or severe delayed flow. Veins showing severe reduction of luminal diameter not associated with deranged flow were not treated [12]. To date, this is probably the most objective and easily reproducible venous protocol described; eliminating assumptions based on subjective findings and complicated venographic scores.

Comment: The contrast emptying time after a specified rate and volume power injection is probably the most practical way to achieve a standardised protocol. Future protocols should probably avoid complex, subjective scores and focus on objective measures. Consider including venous pressure measurements in the venous evaluation protocols.

\section{OTHER TECHNICAL FACTORS}

Other important technical factors include the patient's head position, orthogonal views, and the use of a tilting table.

1. The patient's head position should be either neutral or tilted to the contralateral side being evaluated (this 
latter position opens up the jugular vein and avoids extrinsic compression by surrounding muscles) [6].

2. If venography is the single modality being used, all venograms should include at least two orthogonal views of the site of interest, to better depict the findings [9].

3. The use of a tilting table is appealing to a full evaluation [4]; however, only Traboulsee et al. used a tilting table for venographic evaluation of the patients [4]. This is a potentially cumbersome technique and is not available to most angiographic catheterisation suites. Furthermore, venography in the erect position would probably not evaluate the jugular veins in a proper fashion since these veins are collapsed in the erect position $[4,5]$.

\section{CONCLUSIONS}

Multimodal imaging has been recommended as the ideal approach in the evaluation of patients suspected of having venous obstructions, consistent with CCSVI [11]. Catheter venography is still an essential component in the evaluation of CCSVI. A specific venographic protocol has not been validated; it would be extremely useful to have authors of upcoming reports standardise their venographic protocols. This article addresses the advantages and disadvantages of reported approaches; based on previous venographic protocols reported, we think that the following approach would be a reasonable start:

1. The femoral venous approach is up to the operator's preference and depends on the veins being evaluated.

2. Use of a side hole catheter for contrast injection.

3. Use of diluted contrast medium.

4. Use of automatic, low-pressure power injections with specific rates and volumes of contrast injection.

5. If venography is the single imaging modality used, at least two orthogonal views of the site of interest should be obtained and specified.

6. Specify the image acquisition rates during venography.

7. Use of contrast-emptying time as an objective, easily reproducible parameter to determine the presence of a significant venous obstruction.

8. Until new information is available or validated, a luminal stenosis of greater than $50 \%$ will still be considered as a significant decrease in luminal diameter.

\section{References}

1. Simka M., Hubbard D., Siddiqui A.H., Dake M.D., Sclafani S.J., Al-Omari M, Eisele C.G., Haskal Z.J., Ludyga T., Miloševič Z.V., Sievert H., Stehling M.K., Zapf S., Zorc M.; International Society for Neurovascular Disease. Catheter venography for the assessment of internal jugular veins and azygous vein: position statement by expert panel of the International Society for Neurovascular Disease. Vasa 2013; 42: 168-176.

2. Webb W.R., Gamsu G., Rohlfing B.M. Catheter venography in the superior vena cava syndrome. AJR Am J Roentgenol 1977; 129: 146-148.
3. Gabrielsen T.O., Bookstein J.J. Jugular venography by catheter approach from the arm. Radiology 1968; 91: 378-379.

4. Traboulsee A.L., Knox K.B., Machan L., Zhao Y., Yee I., Rauscher A., Klass D., Szkup P., Otani R., Kopriva D., Lala S., Li D.K., Sadovnick D. Prevalence of extracranial venous narrowing on catheter venography in people with multiple sclerosis, their siblings, and unrelated healthy controls: a blinded, case-control study. Lancet 2014; 383: 138-145.

5. Zamboni P., Galeotti R., Menegatti E., Malagoni A.M., Gianesini S., Bartolomei I., Mascoli F., Salvi F. A prospective open-label study of endovascular treatment of chronic cerebrospinal venous insufficiency. J Vasc Surg 2009; 50: 1348-1358. e1-3.

6. Ferral H., Behrens G., Tumer Y., Riemenschneider M. Endovascular diagnosis and management of chronic cerebrospinal venous insufficiency: retrospective analysis of 30-day morbidity and mortality in 95 consecutive patients. AJR Am J Roentgenol 2013; 200: 1358-1364.

7. Karmon Y., Zivadinov R., Weinstock-Guttman B., Marr K., Valnarov V., Dolic K., Kennedy C.L., Hojnacki D., Carl E.M., Hagemeier J., Hopkins L.N., Levy E.I., Siddiqui A.H. Comparison of intravascular ultrasound with conventional venography for detection of extracranial venous abnormalities indicative of chronic cerebrospinal venous insufficiency. J Vasc Interv Radiol 2013; 24: 1487-1498. e1.

8. Hubbard D., Ponec D., Gooding J., Saxon R., Sauder H., Haacke M. Clinical improvement after extracranial venoplasty in multiple sclerosis. J Vasc Interv Radiol 2012; 23: 1302-1308.

9. Mandato K.D., Hegener P.F., Siskin G.P., Haskal Z.J., Englander M.J., Garla S., Mitchell N., Reutzel L., Doti C. Safety of endovascular treatment of chronic cerebrospinal venous insufficiency: a report of 240 patients with multiple sclerosis. J Vasc Interv Radiol 2012; 23: 55-59.

10. Werner J.D., Siskin G.P., Mandato K., Englander M., Herr A. Review of venous anatomy for venographic interpretation in chronic cerebrospinal venous insufficiency. J Vasc Interv Radiol 2011; 22: 1681-1690.

11. Zivadinov R., Karmon Y., Dolic K., Hagemeier J., Marr K., Valnarov V., Kennedy C.L., Hojnacki D., Carl E.M., Hopkins L.N., Levy E.I., Weinstock-Guttman B., Siddiqui A.H. Multimodal noninvasive and invasive imaging of extracranial venous abnormalities indicative of CCSVI: results of the PREMiSe pilot study. BMC Neurol 2013; 13: 151.

12. Veroux P., Giaquinta A., Perricone D., Lupo L., Gentile F., Virgilio C., Carbonaro A., De Pasquale C., Veroux M. Internal jugular veins out flow in patients with multiple sclerosis:a catheter venography study. J Vasc Interv Radiol 2013; 24: 1790-1797.

13. Raju S. Best management options for chronic iliac vein stenosis and occlusion. J Vasc Surg 2013; 57: 1163-1169.

14. Ludyga T., Kazibudzki M., Simka M., Hartel M., Swierad M., Piegza J., Latacz P., Sedlak L., Tochowicz M. Endovascular treatment for chronic cerebrospinal venous insufficiency: is the procedure safe? Phlebology 2010; 25: 286-295.

15. Sclafani S.J. Intravascular ultrasound in the diagnosis and treatment of chronic cerebrospinal venous insufficiency. Tech Vasc Interv Radiol 2012; 15: 131-143.

16. Zamboni P., Galeotti R., Menegatti E., Malagoni A.M., Tacconi G., Dall'Ara S., Dall'Ara S., Bartolomei I., Salvi F. Chronic cerebrospinal venous insufficiency in patients with multiple sclerosis. J Neurol Neurosurg Psychiatry 2009; 80: 392-399. 